\title{
Compreendendo jogos de computador para uma pessoa como sistemas experimentais
}

\author{
Sebastian Möring \\ Doutor; University of Potsdam, Potsdam, Germany \\ smoering@uni-potsdam.de \\ Tradução: Mayara Caetano \\ Universidade Federal do Rio Grande do Sul, Porto Alegre, RS, Brasil; University of Turku, Turku, Finlândia \\ mayarcae@gmail.com
}

Revisão da Tradução: José Messias

Universidade Federal Fluminense, Niterói, RJ, Brasil

jrmessias.santos@gmail.com

\section{Resumo}

Esse artigo analisa e teoriza as condições que permitem a compreensão do gameplay de jogos de computador single player (single player computer game play ou SPCGP no original) como parte de uma prática de produção de conhecimento chamada experimentação. Portanto, baseia-se na teoria de sistema experimental de Hans-Jörg Rheinberger. Tal abordagem é uma contribuição útil ao campo da hermenêutica dos jogos de computador, a medida que permite compreender o gameplay como um processo hermenêutico, assim como, descreve em maiores detalhes o círculo hermenêutico de gameplay. $\mathrm{O}$ artigo argumenta que, até então, a experimentação nos game studies e na hermenêutica de jogos tem sido equiparada com processos padrões de gameplay. Em outras palavras, todo gameplay é experimentação. Os exemplos, frequentemente mencionados nesses casos são, entretanto, meros dispositivos teste de acordo com a teoria de Rheinberger. A fim de ser um sistema experimental, os jogos de computador precisam ser capazes de produzir inovações reais por si mesmo. Isso, depende por um lado, do nível de complexidade que um determinado jogo exibe, e por outro, do nível de criatividade de dado jogador. Por fim, o artigo sugere que existem apenas quatro casos diferentes nos quais os jogos de computador tornam se geradores reais de surpresas e, portanto, qualificam ou participam de sistemas experimentais que apresentarei e discutirei: glitches em jogos que facilitam as chamadas façanhas em SPCGP; jogos suficientemente complexos, com alto grau de emergência; o 
processo de produção de jogos de computador; e jogos que historicamente participam do avanço da ciência.

\section{Palavras-chave}

Jogos de computador. Single player. Sistemas experimentais.

Hermenêutica. Dispositivo de teste.

\section{Introdução}

Nesse artigo, gostaria de analisar como os jogos de computador e, especificamente, o jogo de computador para um jogador ou individual (single player), podem ser compreendidos como sistemas experimentais que produzem conhecimento devido à sua capacidade de produzir inovações (novelties). Referindo-me à noção de sistema experimental da teoria do conhecimento e das ciências da vida, gostaria de introduzir distinções que permitam diferenciar entre noções de experimentação em jogos de computador ${ }^{1}$ como dispositivos de teste e noções mais avançadas de experimentação em jogos de computador, onde os jogos produzem verdadeiras inovações.

É uma opinião popular que jogar jogos de computador para um jogador (single player computer game play, ou SPCGP) está profundamente entrelaçado com um elemento de experimentação. É até possível dizer que o SPCGP surge principalmente na forma de experimentações se, por exemplo, um jogador inexperiente se envolve com um jogo de computador em particular, como Limbo (Playdead, 2010), pela primeira vez. Para se tornar o "implied player" (AARSETH, 2007) de Limbo, o jogador precisa aprender o que é necessário fazer para manter o jogo em jogo e poder jogá-lo (ver, "game play condition" ou “condição de gameplay" em LEINO, 2009). Desse modo, SPCGP implica práticas de coleta de conhecimento desde o início.

Em sua metodologia de análise de jogos, Espen Aarseth identifica o processo de aprendizagem como uma forma de experimentação. Ele escreve "para progredir pelas fases de aprendizado de um jogo, o jogador precisa explorar várias estratégias e experimentar com diferentes técnicas (2003, p.4 - trad. nossa). No mesmo texto, Aarseth relaciona a experimentação à exploração quando fala dos "experimentos dos exploradores"

\footnotetext{
${ }^{1}$ N.T.:O autor utiliza o termo computer game play. Nesta frase, optou-se por traduzir apenas como jogo de computador. Durante o texto, o autor faz uso constante dos termos game play e computer game play. 0 primeiro será mantido no original e o segundo traduzido como jogo de computador.
} 
(experiments of explorers) (ibdem, p.4). Desse modo, ele classifica as principais atividades do tipo de jogador de Richard Bartle (1996), o explorador, como experimentação. Ao jogar pela primeira vez o simulador de voo de Bruce Artwick, o Designer de Jogos Will Wright também relatou seu envolvimento com o jogo em termos de exploração e experimentação:

\begin{abstract}
A primeira coisa que fiz foi entrar e começar a explorar o comportamento do espaço. Experimentando todas as coisas diferentes com o avião. 0 que acontece se eu for direto para cima? Até onde posso ir? 0 que acontece se eu bater? 0 que acontece se eu fizer isso, aquilo e aquilo outro? Então, eu pude realizar experimentos nesse mundo. E ao desempenhar esses experimentos, consegui ter uma percepção mais acurada sobre qual era o modelo interno" (apud PEARCE, 2002).
\end{abstract}

Wright utiliza ambas as expressões - exploração e experimentação - para descrever o seu processo de game play como se fossem intercambiáveis ou pelo menos indispensáveis. Esse elemento de exploração também está contido na noção de Aarseth do jogo de computador como trabalho ergódico, onde o ergódico é um termo híbrido do Grego hodos"(caminho) e ergon (trabalho). (AARSETH, 1997, p.1). No jogo de computador, esse elemento de descoberta com alguma frequência consiste literalmente em uma forma de deslocamento (vide o significado de exploration, em inglês, no Oxford English Dictionary Online 2016). Particularmente, em jogos de computador em primeira pessoa, o personagem do jogador literalmente precisa ser movido através do espaço e, frequentemente, para áreas que eram previamente desconhecidas do jogador. De modo semelhante, o processo de pesquisa científica é frequentemente descrito metaforicamente em termos de exploração, na medida em que um pesquisador viaja para uma "área desconhecida" de seu "campo" para descobrir novos caminhos. Assim, Wright compara o processo de game play com o de "experimentação científica”. (apud PEARCE, 2002)

0 historiador da ciência alemão, Hans-Jörg Rheinberger, investiga como pesquisadores em ciências da vida realmente descobrem novidades/inovações sobre suas áreas de atuação. Descrevendo a estrutura da descoberta científica, Rheinberger usa o termo "sistema experimental" (RHEINBERGER, 1997, p.1) e ainda conecta esse processo ao de jogar um jogo. Com François Jacob, ele fala do sistema experimental como um jogo de possibilidades. (“jeu des possibles”, c.f. RHEINBERGER, 1997, p.76)

Ainda que, jogo de computador seja definido com frequência como uma experimentação e até a experimentação científica seja comparada ao game play, há motivos para supor que nem todos os tipos de SPCGP permitem ser chamados por experimentação. A 
teoria de sistemas experimentais de Rheinberger, por exemplo, distingue sistemas experimentais de dispositivos de teste. Portanto, a questão central para esse trabalho é: quais condições precisam ser satisfeitas para considerar jogos de computador para um jogador como sistemas experimentais ao seguir a teoria do conhecimento de Rheinberger?

Essa questão irá provocar respostas que são cruciais para o campo da hermenêutica nos jogos. Um dos primeiros a sugerir esse campo é Aarseth quando ele identifica o elemento da coleta de conhecimento nos SPCGP com destaque como "hermenêutica em tempo real" (real-time hermeneutics)". (AARSETH, 2003, p.5) A seguir, essa ideia tornou-se central para o discurso na filosofia dos jogos de computador, que se preocupa com as condições de significado e interpretação dos jogos de computador e é apelidada de "hermenêutica dos jogos". (ARSENAULT e PERRON, 2009; LEINO, 2010; 2012; ARJORANTA, 2011; 2015; KARHULAHTI, 2012; 2015; MÖRING, 2013; VELLA, 2015) Essa linha de raciocínio implica que a apreensão de um certo tipo de conhecimento aumenta a compreensão de como um jogo de computador é e pode ser jogado.

Para responder à questão de pesquisa mencionada acima, vou me embasar no conceito de sistema experimental de Rheinberger. Irei trabalhar a ideia de game play, uma vez que experimentar o jogo Limbo (Playdead 2010) supostamente consiste num game play experimental. Entretanto, uma breve análise demonstra que, o jogo consiste principalmente em encontrar as ditas "coisas ocultas" (hidden things) em vez de verdadeiras inovações. Consequentemente, será argumentado que os jogos requerem certas condições para se qualificarem como potenciais sistemas experimentais. Esses são, por um lado, jogos com certa complexidade e abertura que permitem encontrar inovações no game play e, por outro lado, um certo tipo de jogador que opera com um estilo inovador específico de game play. Essa distinção, além disso, permite diferenciar entre jogar um jogo e jogar com um jogo. (LEINO, 2010, p. 141) 0 último se qualifica como uma atividade experimental e, mais ainda, como uma atividade comum de game play. Eventualmente, discutirei quatro casos que diferem em grau em que os jogos de computador são sistemas experimentais ou participam em sistemas de experiência. Por fim, concluirei que a experimentação acontece em jogos em níveis diferentes, incluindo o processo de desenvolvimento de jogos e o Jogo de Guerra Prussiano (Prussian Kriegsspiel), que ajudou a desenvolver o conhecimento em matemática através da experimentação. 


\section{Sistema Experimental de Rheinberger}

Devo começar apresentado o sistema experimental de Rheinberger. 0 filósofo, biólogo e historiador da ciência Hans-Jörg Rheinberger desenvolveu o conceito de sistema experimental (Rheinberger, 1997) enquanto observava cientistas da vida do campo da "biomedicina, bioquímica e biologia molecular" (RHEINBERGER, 1998, p. 427). Apesar de estabelecido no contexto das ciências naturais, seu conceito de sistemas experimentais é amplamente aplicado nas humanidades também. Sistemas experimentais diferem de uma compreensão de senso comum dos experimentos. No senso comum, experimentos são considerados como uma "únicas, instâncias empíricas bem definidas envolvidas na elaboração de uma teoria e performadas com a função de corroborar ou refutar certas hipóteses." (RHEINBERGER, 1997, p. 27). Para dar um exemplo do campo dos jogos de computador: quando Will Wright diz que jogar com o simulador de voo Artwick é "como um processo científico. É como um 'experimento hipotético, mude suas hipóteses' tipo de ciclo que estava ocorrendo" (apud, PEARCE, 2002), ele usa essa compreensão comum sobre experimentação como se o processo de jogar fosse um "teste de hipóteses" ou uma "atividade dirigida pela teórica" (RHEINBERGER, 1997, p.27) na qual práxis é subordinada à teoria. Ainda assim, há pelo menos dois problemas com a noção comum sobre experimentos. Um resulta do ponto de vista da história da ciência, e outro do ponto de vista do jogo de computador.

Rheinberger se refere ao biólogo e físico polonês Ludwik Fleck, que introduziu o termo "sistema de experimentos". (FLECK apud RHEINBERGER, 1997, p. 27, grifos do autor) De acordo com Fleck, um único experimento não pode provar uma teoria; consequentemente, pesquisadores não lidam com "experimentos isolados em relação a uma teoria". (FLECK apud RHEINBERGER, 1997, p. 27) Ao invés disso, "é necessário um completo sistema de experimentos e de controles, configurado de acordo com a suposição ou estilo e realizado por um especialista". (FLECK apud RHEINBERGER, 1997, p. 27 - grifos do autor) Além disso, cientistas experimentais frequentemente não trabalham com experimentos de pesquisa bem definidos cujos resultados são conhecidos previamente, mas eles trabalham com "sistemas de experimentos que normalmente não são bem definidos, e não provêm respostas claras". (FLECK apud RHEINBERGER, 1997, p. 27)

Do ponto de vista do game play há um problema relativo à primazia da teoria sobre a práxis. Game play, compreendido como "as ações, estratégias e motivações dos jogadores" 
(AARSETH, 2003, p. 2) em um jogo de computador, não é necessariamente uma atividade direcionada pela teoria em termos de provar, ou julgar falsas, hipóteses teóricas testando os efeitos de diferentes ações. Uma vez que é em grande medida sobre as ações dos jogadores, o game play deve ser compreendido também como uma atividade muito prática, o que requer, portanto, uma hermenêutica prática ao invés de uma hermenêutica teórica (textual) (MÖRING, 2014, p. 227-315; KARHULAHTI, 2012) Especialmente os jogos de ritmo acelerado às vezes deixam pouco ou nenhum tempo para construir hipóteses no pensamento consciente. A construção de hipóteses ocorre antes durante a fase de envolvimento macro do jogo de computador, que descreve o engajamento do jogador em momentos onde ele não está realmente operando o jogo e compondo estratégias e táticas. (CALLEJA, 2011, p. 40) Contrariando a ideia comum de experimentação, o game play não começa necessariamente com a teoria, mas deve ser pensado como um ciclo (ARSENAULT e PERRON, 2009) com fases intercambiáveis da práxis e da teoria em termos iguais. 0 mesmo pode ser dito para o conceito de sistemas experimentais de Rheinberger que refuta "uma perspectiva da filosofia da ciência que coloca a teoria em primeiro lugar" (RHEINBERGER, 1997, p. 26).

Empiricamente, os sistemas experimentais são sempre determinados por seus contextos. As instituições nas quais eles ocorrem, as pessoas que trabalham neles, o conhecimento pré-existente, a acessibilidade ao conhecimento, etc. influenciam como um sistema experimental funciona. No nível estrutural, os sistemas experimentais consistem em uma construção necessariamente dupla: uma coisa epistêmica e algo chamado objeto técnico que são "diferente, porém inseparáveis". (RHEINBERGER, 1997, p.28) Uma coisa epistêmica é "uma entidade cujas características desconhecidas são o objetivo de uma investigação experimental". (RHEINBERGER, 1997, p. 238). Uma característica particular desse objeto científico é sua "imprecisão" (vagueness) implícita (RHEINBERGER, 1997, p. 238), pois incorpora o desconhecido. Essa imprecisão se origina do paradoxo de que as coisas epistêmicas estão ausentes enquanto elas estão ao mesmo tempo presentes nos experimentos. (RHEINBERGER, 1997, p.238). Objetos técnicos, por outro lado, pertencem às condições experimentais e a "um campo mais amplo de práticas epistêmicas e culturas materiais, incluindo instrumentos, dispositivos de inscrição, modelo[s] [...]. e os teoremas flutuantes ou conceitos delimitantes ligados a eles". (RHEINBERGER, 1997, p. 29) A relação entre a coisa epistêmica e o objeto técnico pode ser descrita como complementar; uma vez que objetos técnicos “'contêm' os objetos científicos no duplo sentido dessa expressão: eles 
os incorporam, e através desse mesmo acolhimento, os restringem e os limitam.". (RHEINBERGER, 1997, p. 29) Por exemplo, um microscópio como um objeto técnico já determina por causa de sua materialidade e sua lógica operacional as "possíveis representações de uma coisa epistêmica" (RHEINBERGER, 1997, p. 29) antes mesmo de qualquer descoberta ao microscópio. 0 mesmo pode ser dito sobre jogos de computador. Tudo o que é conhecido sobre a lógica de um jogo é sempre determinado pelas mecânicas do jogo, a interface, o código do jogo etc. Rheinberger observa que os "objetos técnicos incorporam o conhecimento de um determinado campo de pesquisa em um determinado período". (RHEINBERGER, 1997, p. 245) Ambas, coisas epistêmicas e objetos técnicos, precisam ser vistos como dois extremos de um continuum que permite "todos os níveis possíveis de híbridos" (RHEINBERGER, 1997, p. 30) e coisas epistêmicas podem, se suficientemente estabilizadas, "transformar-se no repertório técnico do arranjo experimental”. (RHEINBERGER, 1997, p. 29) O microscópio deve, então, ser considerado como o resultado ou a coisa empírica de um sistema experimental precedente, antes de participar como um objeto técnico em outro sistema experimental.

\section{Limbo: Entre Sistema Experimental e Dispositivo de Teste}

Pode-se exemplificar e testar o conceito de sistema experimental e seus componentes (coisas epistêmicas e objetos técnicos) com o game play do jogo Limbo da desenvolvedora baseada em Copenhagen Playdead (2010). Escolhi Limbo porque é, de um lado, simples o suficiente para servir como um exemplo esclarecedor. Por outro lado, ele permite a problematização de diferenças entre noções do senso comum de experimentos e de sistemas experimentais quando se trata de SPCGP. Embora esteja claro que o processo de game play de Limbo suporta a noção do senso comum de experimentos, parto da hipótese de que Limbo pode ser compreendido como um sistema experimental porque a mecânica de jogo exige que o jogador experimente com o jogo com a finalidade de cumprir as condições de game play do jogo. (LEINO, 2009)

Bem no começo de Limbo, o avatar desperta em um gramado em uma floresta 2D muito escura e em cores monocromáticas. 0 mundo de jogo e o personagem do jogador são as únicas silhuetas visíveis e, por esse motivo, há dificuldade em reconhecê-los a princípio. Nessa cena inicial na floresta, não há nenhuma informação sobre por motivo pelo qual o 
avatar desperta, por que ele está na floresta, o que ele deveria fazer e assim por diante. Apenas no menu, o jogador pode acessar informações muito básicas sobre atividades possíveis que o avatar pode performar (movimentar-se para esquerda e direita, pular, interagir com objetos). Essas atividades podem ser consideradas como objetos técnicos que delimitam o espaço de ações possíveis no mundo e com tal, o "espaço de possibilidades" de um jogo. (SALEN e ZIMMERMAN, 2004, p.66-67) Elas, simultaneamente, moldam indiretamente qualquer novo estilo de jogo que possa ser descoberto.

O principal objetivo de jogo deveria ser encontrar a coisa epistêmica, o objeto do conhecimento que ainda não está à disposição do jogador. Pode-se pensar que a primeira coisa epistêmica do jogo Limbo é descobrir “O que o avatar deveria fazer no mundo?" Esse conhecimento, contudo, já está disponível para os jogadores que possuem alguma experiência com o gênero de jogos de plataforma. Como Limbo é um jogo de plataforma bidimensional, conhecimento prévio sobre como jogar jogos de plataforma está disponível para maioria dos jogadores. Portanto, é mais provável que o jogo seja abordado de acordo. Mais problemáticos são os numerosos obstáculos perigosos (perigosas serras circulares, fendas/desfiladeiros enormes, organismos prejudiciais, etc.) ao longo do caminho, que é frequentemente irreconhecível à primeira vista e que requer que o jogador encontre maneiras de superá-los. Usando uma abordagem passo-a-passo tentativa-e-erro, o jogador aprende como evitar um fim prematuro do jogo e como progredir. A coisa epistêmica como superar os obstáculos - moldam apenas em retrospecto, depois que o avatar morre várias vezes e a solução torna-se mais clara a cada tentativa. Rheinberger escreve de modo similar que "coisas epistêmicas [...] são recursivamente constituídas e assim intrinsicamente coisas históricas". (RHEINBERGER, 1997, p. 76) No curso de uma sessão de game play, algumas das coisas epistêmicas, como o uso de uma caixa em Limbo, tornam-se objetos técnicos à medida que eles se tornam conhecimento suficientemente estabilizado. Eles tornam-se um método que é repetidamente reprodutível. (RHEINBERGER, 1997, p. 30)

Limbo se constrói com conhecimentos e habilidades que são sucessivamente acumuladas através do game play. No entanto, o jogo apresenta o jogador constantemente com novos desafios como, por exemplo, uma repentina modificação de gravidade em um estágio posterior do jogo. Alguns críticos, portanto, objetam que o jogo não suporta um processo de aprendizado incremental: "Ao invés de pedir para que você aplique o que aprendeu a partir de suas mortes anteriores, o jogo continua modificando as regras, para 
então poder matá-lo novamente. É como se estivesse inventando coisas, como um primeiro rascunho que poderia usar uma boa revisão". (KRPATA, 2010)

Apesar de seus desafios, Limbo não é uma exceção real de outros jogos de computador que suportam uma experiência de aprendizado mais linear. A interação de encontrar uma coisa epistêmica e transformá-la em um objeto técnico no processo de game play é a base de muitos outros jogos de computador também.

Entretanto, o caráter passo-a-passo de tentativa-e-erro do game play de Limbo leva a outro ponto crítico. Uma estratégia tentativa-e-erro implica a existência de uma solução correta que precisa meramente ser descoberta. Por isso, pode-se argumentar que o game play de Limbo não lida exatamente com coisas epistêmicas, mas sim com o descobrimento de "coisas ocultas". (RHEINBERGER, 1997, p.28) Se, descobre-se que o game play de Limbo era apenas sobre a descoberta de coisas ocultas, então não se qualificaria como sistema experimental. Rheinberger escreve, portanto, que as coisas epistêmicas: "não são apenas coisas escondidas a serem trazidas para a luz através de manipulações sofisticadas". (RHEINBERGER, 1997, p. 28) Essa descrição, entretanto, caracteriza o game play de Limbo muito apropriadamente. Pode-se argumentar que as alegadas coisa epistêmica em jogo que são descobertas quando um jogador começa a jogar o jogo e a explorar sua condição de game play são, de fato coisas ocultas. Seguindo Will Wringht, o jogador tenta uma "fazer a engenharia reversa da simulação" ("reverse engineer the simulation") (apud PEARCE, 2002) em tais casos. Coisas escondidas são características de "dispositivo[s] de teste" ("testing device[s]"). (RHEINBERGER, 1998, p. 291) No entanto, enquanto coisas epistêmicas são verdadeiramente desconhecidas, as coisas ocultas são características de mecânicas já conhecidas e estabilizadas. (RHEINBERGER, 1997, p. 291) No caso de Limbo, os métodos a serem descobertos por um jogador foram escondidos de antemão pelos designers do jogo. Esses são destinados a serem descobertos mais cedo ou mais tarde. Embora se possa argumentar que as coisas ocultas são, pelo menos, novas para jogador que acaba de descobri-las, a ideia de Rheinberger de inovação através de sistemas de experimentação referem-se ao conhecimento que não era conhecido por ninguém. Consequentemente, os sistemas de experimentação são capazes de produzir inovações, são "um jogo de inovação" (“a game of innovation"). (RHEINBERGER, 1997, p. 31)

Game designers que tendem a exibir grande controle autoral, também tendem a desenvolver jogos de um modo que é rígido o suficiente para exibir esse controle. Ainda, se um jogo é "muito rígido", e produz apenas "padrões ou réplicas" ele é um dispositivo de 
teste. (RHEINBERGER, 1997, p. 291) Esse é certamente o caso de Limbo: ele limitou as affordances do mundo de jogo (LINDEROTH, 2013) e as habilidades limitadas do avatar não permitem muitas estratégias alternativas e para cada obstáculo geralmente há apenas uma solução para superá-lo. Portanto, Limbo deve ser considerado um dispositivo de teste ao invés de um sistema experimental. De acordo com Rheinberger, para Limbo tornar-se um sistema experimental ele "deve ser capaz de reprodução diferencial, a fim de comportar-se como um dispositivo para produzir coisas epistêmicas cuja possibilidade está além do nosso conhecimento atual, isso é, para comportar-se como um 'gerador de surpresas'". (RHEINBERGER, 1998, p. 287) A distinção entre dispositivos de teste e sistemas experimentais é importante, pois os dispositivos de teste limitam enormemente o espaço de possibilidade. A noção de dispositivo de teste é reminiscente da qualidade do jogo que é descrita como "o mesmo, porém diferente" ("same but different") por Katie Salen e Eric Zimmerman:

Jogos possuem uma qualidade que chamamos de mesmo, porém diferente. Todas as vezes que jogamos um jogo, a estrutura formal permanece a mesma, mas o modo como as regras funcionam são diferentes. Essa qualidade dos jogos o torna prazerosa para que os jogadores explorarem 0 espaço de possibilidades" (SALEN e ZIMMERMAN, 2004, p. 361).

Pode-se dizer que os sistemas experimentais, assim como os jogos, implicam o mesmo tipo de reprodução diferencial. Entretanto, pode-se argumentar que, no caso de sistemas experimentais, o aspecto da diferença está em primeiro plano, pois seu propósito é produzir inovações. No caso de um jogo como Limbo, a atualização de diferentes possibilidades por espaço de possibilidades do jogo é conhecida por concretizar possibilidades que foram conhecidas antes.

A questão que segue disso é: quais condições são necessárias para um jogo produzir inovações reais? Quais condições devem ser preenchidas para que um jogo seja considerado um sistema experimental ao invés de um dispositivo de teste? A argumentação seguinte baseia-se no pressuposto que pelo menos dois elementos são necessários para um jogo ser um sistema experimental em potencial. Por um lado, ele precisa de um tipo específico de jogador e de um certo estilo de game play para ser considerado um sistema experimental. Por outro lado, o próprio artefato de jogo deve ser capaz de gerar surpresas e reproduzir diferenciação. Portanto, a secção seguinte argumenta que um jogo requer certa abertura e complexidade para participar de um sistema experimental. 


\section{Jogos Abertos de Alta Complexidade}

Nessa secção, argumento que os jogos de computador precisam ser suficientemente abertos e complexos a fim de serem considerados sistemas experimentais. Jesper Juul introduziu uma proeminente distinção entre jogos de progressão e jogos de emergência para o estudo de jogos de computador. (JULL, 2002, p.324) Jogos de progressão são jogos nos quais "o jogador tem que desempenhar um conjunto de ações pré-definidas a fim de finalizar o jogo" e eles se prestam a um forte controle autoral. (ibidem) A respeito do conceito de sistema experimental, jogos de progressão como Limbo parecem ser casos claros de dispositivos de teste. Os jogos de emergência, por outro lado, são caracterizados por um "número menor de regras que combinam e grande número de variações de game", por isso, eles são com frequência atribuídos com um grande valor de rejogabilidade do que jogos de progressão. (JUUL, 2002, p. 324)

A distinção de Juul entre jogos de emergência e jogos de progressão é certamente discutível. Ambos os tipos de jogo não são distinguidos pela presença ou ausência de um elemento de emergência, mas sim por pelo nível de complexidade da emergência envolvido. O cientista da computação e desenvolvedor de software Jochen Fromm desenvolveu uma taxonomia de quatro tipos de emergência: Tipo 1 emergência simples intencional; Tipo 2 emergência fraca; Tipo 3 emergência múltipla; e Tipo 4 emergência forte. (FROMM, 2005)

0 jogo de progressão de Juul é igual à forma menos complexa de emergência: Tipo 1a simples/nominal emergência sem feedback top-down e emergência intencional simples. (FROMM, 2005) Esse tipo de emergência é comparável "ao design intencional de uma máquina" com papéis específicos e fixos para cada elemento, que nunca mudam e são sempre os mesmos. (FROMM, 2005) Fromm compara isso as engrenagens de um relógio. Por isso, nos jogos de progressão, o designer controla o espaço de possibilidades do jogo ao mantendo-o limitado. A emergência do Tipo 4 representa o mais alto grau de complexidade. Exemplos para emergência do Tipo 4 são a vida e a cultura. (FROMM, 2005) Os jogos de computador que exemplificam esse tipo de emergência são, talvez, jogos massivos multiplayer online. Esses jogos desenvolvem suas próprias regras de comportamento e práticas culturais, bem como os sistemas econômicos e políticos, além da materialidade codificada dos jogos de computador, como Eve Online (CCP Games, 2003). Ao contrário dos jogos de computador para um jogador, os jogos massivos multiplayer online podem ser 
considerados culturas por direito próprio. No caso de Eve Online, o jogo cresceu tanto e tornou-se tão complexo que até mesmo aquelas mídias online, que não são dedicadas exclusivamente a notícias vindas do campo dos jogos de computador, reportam sobre acontecimentos dentro do jogo. 0 canal de mídia e tecnologia The Verge reportou:

\begin{abstract}
“EVE Online possui sua própria economia, política e sistema de negócios, construído quase que inteiramente pelos jogadores nos 10 anos que o jogo tem corrido. Ele também possui suas próprias guerras, uma vez que grandes alianças disputam pelo controle espacial nesse jogo massivo multiplayer online. Um desses conflitos veio a frente ontem na maior batalha no jogo nos dez anos de história. [...] 0 dano resultante foi avaliado em mais de $\$ 200.000$ em moeda do mundo real.". (MCCORMICK, 2014).
\end{abstract}

A observação de Juul que: "muitos jogos podem ser encontrados em uma escala entre emergência e progressão" (ibdem, 2005, p. 71) pode ser reformulada para "muitos jogos podem ser encontrados numa escala entre menos e maior complexidade". Sem aprofundar a teoria dos sistemas complexos e os diferentes tipos de complexidade pode-se concluir que os jogos com maior grau de complexidade possuem melhor potencial para a produção de inovação e surpresas. Isso os qualifica como um sistema experimental em potencial, à medida que se tornam cada vez menos previsíveis quanto maior o seu grau de emergência (1 para 4). 0 grau de emergência e imprevisibilidade é semelhante ao grau de abertura de um sistema ou jogo:

"Um jogo complexo como Civilization, Deus Ex ou GTA 3 pode ser vencido [zerado] numa questão de dias ou semanas, mas devido a amplitude da simulação e da coletiva ingenuidade dos jogadores, o potencial para novas descobertas é infinito". (AARSETH, 2003, p. 3).

Consequentemente, os jogos abertos nesse sentido permitem que novas estratégias e táticas sejam descobertas e desenvolvidas. Nas seguintes novas estratégias podem então mudar de um estado inicial de uma coisa epistêmica para uma "híbrida intermediária" ("halfway-hibrid") e depois, quando suficientemente estabilizada, para um objeto técnico ou um "método". (RHEINBERGER, 1997, p. 30) Esse método pode então evoluir para um modo padrão de como um jogo específico é jogado. Por exemplo, entre as diversas estratégias de abertura do xadrez, algumas podem ser consideradas suficientemente estabilizadas. Aqueles pertencem ao repertório padrão de jogar xadrez.

Especialmente "falhas imprevisíveis e erros inesperados no sistema de software e hardware" que "são uma especial e indesejada forma de emergência" (FROMM, 2005) privilegiam os respectivos jogos como potenciais sistemas experimentais, pois permitem 
que surjam surpresas não intencionais durante o game play. Rheinberger argumenta que os sistemas experimentais são produtivos especificamente se não estiverem bem definidos. Isso contrasta com a ideia clássica de experimentos como construções bem definidas para falsificar ou provar hipóteses. (RHEINBERGER, 1997, p. 27) Portanto, é possível distinguir entre intenção não intencional que ocorre em jogos complexos e não intencionalidade. 0 primeiro ocorre em jogos que não são bem definidos na forma como os dispositivos de teste são bem definidos, mas esses jogos são bem definidos no sentido de possibilitarem um espaço de possibilidades que é suficientemente aberto para permitir que surjam surpresas em vez de sendo rigidamente limitante e previsível. Os últimos dizem respeito a bugs ou glitches. Ambos os tipos de surpresas podem ser explorados experimentalmente.

Nesse sentido, bugs menores ou maiores apresentam uma lacuna ou uma "abertura" literal na estrutura do um jogo, que pode ser explorada pelos jogadores para inventar novas estratégias. Para dar um exemplo, deixe-me considerar o bem conhecido truque de rocketjumping em Quake III Arena (id Software, 1999) como descrito por Jesper Juul: "rocketjumping [...] é uma tática de pular no ar, disparando um foguete para o chão que está abaixo, e voando na onda de choque da explosão. (Essa é uma forma de pular mais longe do que você poderia ser capaz)". (ibidem, 2005, p. 81) Essa possibilidade é explorada como uma tática no jogo e fornece vantagens significantes para jogar o jogo com sucesso ao longo de um estilo de jogo em que essa tática é ausente. Entretanto, assim que o rocket-jumping torna-se uma tática padrão, ela se torna um método estabilizado (coisa técnica) e uma forma padrão de jogar Quake III Arena. Para dizer isso com Heidegger: "rocket-jumping não será mais uma forma autêntica de game play, mas se tornará inautêntica". (HEIDEGGER, 2008; cf. LEINO e MÖRING, 2015) Com Vilém Flusser pode-se dizer que esse truque descobriu partes da "competência de game play" ("Spielkompetenz", a soma de todas as possibilidades de game play uma estrutura de jogo potencialmente contida) e uma expansão do "universo de game play" ("Spieluniversum", a competência da estrutura de jogo já realizada). (FLUSSER, 1998, p.330)

Inovações ou surpresas podem ocorrer de duas maneiras dependendo do tipo de jogo. Aqui a distinção de Flusser entre jogos abertos e fechados é útil. (ibidem, 1998, p.330-331) Em jogos abertos, estratégias novas de jogo podem ser implementadas no jogo sem afetar o código ou a materialidade do jogo. (cf. LEINO, 2015) em jogos fechados novos elementos de jogo só podem ser implementados no jogo quando modificam a materialidade do jogo por exemplo através de modificações no software ou hardware. (cf. GALLOWAY, 2006) Jogos 
abertos nesse sentido são jogos populares como Minecraft (Mojang, 2011), Kerbal Space Program (Squad, 2011), e Space Engineers (Keen Software House, 2013) Portanto, esses jogos são frequentemente referidos como jogos de mundo aberto. Por exemplo, Minecraft permite que elevadores e computadores funcionais de 16-bits sejam construídos dentro do jogo sem requererem modificações no software do jogo. Assim, ambos, artefatos de jogo aberto e fechado, podem conter possivelmente bugs e glitches que podem ser explorados. Nesses casos, a não intencionalidade das inovações é intencional e, por isso, está implícita na estrutura do jogo e precisa ser descoberta de algum modo (cf. Rocket-jumping). Ou, as inovações são possíveis ao operar na própria estrutura do jogo. Isso implicaria o nível de game design e, é claro, o nível de modificação no jogo em que o jogador opera no limiar entre game play e game design. Consequentemente, o aspecto diferencial de reprodução no sistema de experimentação pode ser encontrado em jogos que permite inventar novas estratégias de como jogar um jogo no espaço de possibilidades já disponível ou o código de jogo é modificado e, desse modo, abre para novas possibilidades de espaço no nível do game play.

Resumindo, pode-se diferenciar entre jogos fechados com um surgimento muito fraco e alta rigidez de jogos com forte emergência e pouca rigidez. Os primeiros são dispositivos de teste em vez de sistemas experimentais em potencial no nível do game play. Esses jogos só podem ser considerados como sistemas experimentais em potencial no nível do código de jogo devido a modificações no jogo ou devido a por bugs e glitches. Jogos abertos com emergência mais forte podem ser sistemas experimentais em potencial no nível do game play assim como no nível da estrutura de jogo modificável. Por isso, para os jogos, a fim de ser sistemas experimentais, esses requerem adicionalmente um certo tipo de jogador ou um certo tipo de jogo, uma vez que essas inovações, não importa se no nível do código de jogo ou no nível do jogo, precisam ser descobertos por alguém.

\section{Jogador Inovador}

Para que um jogo seja considerado um sistema experimental, é necessário também olhar para aqueles que jogam jogos e que possivelmente são capazes de operar os jogos de um modo que inovações sejam descobertas. Seguindo os tipos de jogador de Richard Bartle, pode-se argumentar que, para um jogo ser um sistema experimental, é necessário um 
jogador que seja simultaneamente um empreendedor avançado, matador, socializador e explorador. (BARTLE, 1996) Todos esses tipos estão, de alguma forma, interconectados e, portanto, todos podem ser importantes para revelar inovações e surpresas. Por exemplo, descobrir sobre a exploração do rocket-jumping requer que um jogador de Quake III Arena sejam um realizador, um matador, um socializador $e$ um explorador. Parece que o tipo explorador é o mais importante nesse exemplo, uma vez que exploradores podem ser considerados os "experimentadores" entre os jogadores: "Os exploradores se deliciam em fazer com que o jogo exponha suas maquinações internas a eles. Eles tentam ações progressivamente esotéricas em lugares selvagens e afastados, procurando por características interessantes (por exemplo, [sic!] Bugs) e descobrindo como as coisas funcionam". (BARTLE, 1996) Por exemplo, um explorador todas as outras atividades de jogo são subordinadas à exploração do funcionamento interno de um jogo.

Parece que os quatro tipos de jogadores de Bartle são um requisito necessários, porém não suficientes, para que um jogo seja um sistema experimental. Rheinberger destaca o nível de experiência do experimentador: "quanto mais ele ou ela [o experimentador] aprende a lidar com seu próprio sistema experimental, mas ele desempenha suas próprias capacidades intrínsecas".. (RHEINBERGER, 1997, p. 24) Aarseth (2003) menciona sete tipos de game play que refletem diferentes graus da experiência de jogar jogos de computadores. Eles descrevem um continuum de muito pouco entendimento a uma grande compreensão sobre como um jogo pode ser jogado: 1) jogo superficial, 2) jogo leve, 3) finalização parcial, 4) finalização total, 5) jogada repetido, 6) jogo especializado e 7) jogada inovador. (AARSETH, 2003, p.6) Claramente, para os jogos serem sistemas experimentais, o jogado inovador é a forma mais significativa de jogo.

A fim de considerar o jogo de computador como parte de um sistema experimental, ele precisa ser capaz de gerar surpresas, assim como o sistema experimental de Rheinberger produz "inovações científicas" e é um "gerador [...] de surpresas". (RHEINBERGER, 1997, p.3) Defendendo, assim, que os tipos de jogo de Aarseth, desde o jogo superficial até o jogo especialista, focam-se em explorar coisas ocultas para diferentes graus de sofisticação, o que também representa diferentes graus de compreensão do game play. Isso implica todas as atividades de exploração que todos os tipos de jogadores não-exploradores de Bartle fazem ao lado de seus objetivos reais também. Como tal, eles podem ser considerados atividades de game play em diferentes níveis. 0 jogador inovador, entretanto, da um passo significativo adiante. Aarseth escreve, o game play inovador "é visto quando os jogadores inventam 
estratégias totalmente novas e jogam o jogo não para ganhar, mas para atingir um objetivo por meios que não são previamente reconhecidos como tal por outros jogadores. (ibidem, 2003 , p. 6) Portanto, o game play inovador requer que o jogador passe por todos os outros estágios da experiência de game play, de obter a experiência essencial para que um sistema experimental funcione como tal. Rheinberger marca isso como uma "qualidade intelectual" ("intelectual quality") e um tipo de "intuição adquirida" ("acquired intuition") ou "conhecimento tácito" ("tacit knowledge"). (RHEINBERGER, 1997, p. 77) Então, o jogador inovador lida com a condição de game play intuitivamente ou tacitamente e pode perseguir seus próprios projetos em relação ao jogo sem estar preso a lidar apenas com sua condição de game play ou com melhorar sua experiência ao lidar com ela.

Anteriormente, eu e Olli Leino nos referimos a isso como game play autêntico, a forma mais elevada de expertise de game play que um jogador pode realizar desde que consegue transcender a condição de game play, pois seu objetivo não é mais satisfazer essa condição, mas sim trabalhar em seu próprio interesse. (LEINO e MÖRING, 2015) Seguindo a leitura de Dreyfus sobre Heidegger (DREYFUS, 1991, p. 26), distinguimos entre três graus nos quais um artefato de jogo pode ser encontrado por um jogador 1) no nível da materialidade, 2) no nível do jogo, e 3) o nível-meta da materialidade/jogo. 0 primeiro nível é derivado do "modo indiferenciado" ("undifferentiated mode") (DREYFUS, 1991, p.26) do ser-no-mundo. Portanto, similar a um recém-nascido o jogador é jogado em um jogo, mas simplesmente encontra sua materialidade ou software ao tentar operá-lo e fazê-lo funcionar contra a resistência da condição de game play. 0 segundo nível se refere ao inautêntico ser-nomundo. Isso significa que o jogador joga um jogo como é normalmente jogado, incluindo regras de habilidades padrão. (SUITS, 2005, p.31) Nesse nível, ela pode dominar o jogo até um ponto em que a condição de game play não é mais percebida como resistência. Ainda assim, ela está jogando o jogo de uma maneira não autêntica ou de "modo renegado"/ "disowned mode" (DREYFUS, 1991, p. 26) Ela não se percebe, mas apenas possibilidades que já foram reveladas. Nesse modo, o jogador encontra o objeto de jogo como jogo. Apenas o terceiro nível se assemelha a um game play autêntico e ao "modo próprio"/ "owned mode" (DREYFUS, 1991, p. 26) do ser-no-mundo. Nesse modo, o jogador não apenas domina o jogo, porém também transcende o jogo jogando de formas que não haviam sido descobertas antes. Nesse nível, o jogador encontra o jogo tanto como material quando como jogo. Porém, ao contrário dos dois primeiros modos, o jogador tem a liberdade de decidir em qual modo ela enfrenta o jogo. Minecraft é jogado no modo indiferenciado quando o jogador acaba de 
encontrar o jogo pela primeira vez a partir de qualquer lugar no mundo do jogo para o qual seu avatar foi literalmente lançado (também conhecido como spawned ou desovado) para. No modo inautêntico, o jogador descobre diferentes maneiras de se envolver com a condição de game play, como nivelar o personagem, matar multidões, aumentar as plantações, etc e quase que literalmente faz o que se faz quando se está no modo sobrevivência do Minecraft até o nível de maestria. 0 modo autêntico de estar em Minecraft significa, então, "transcender a condição de game play e o ser-em-Minecraft inautêntico cotidiano" e "jogar o modo de sobrevivência como se fosse o modo criativo". (LEINO e MÖRING, 2015).

Como sugerido anteriormente, Leino distingue dois modos de game play que ele chama de "jogar um jogo"/"playing a game" e "jogar com um jogo"/"playing with the game". (LEINO, 2010, p.141) Eles se assemelham ao segundo e terceiro nível de modelo tríadico de game play que acabamos de descrever. Jogar um jogo significa jogar de acordo com sua "condição de game play". (LEINO, 2010, p. 133) Por exemplo, em Minecraft (MOJANG, 2011) isso significa evitar um fim prematuro ao deixar o avatar sem morto. 0 sucesso do jogador nesse esforço é avaliado pelo jogo. Imagine, um jogo de tiro onde o avatar do jogador está sob fogo constante. Em tal jogo, o jogador não pode simplesmente fazer o que ele quer, mas é bastante limitado pela materialidade do jogo que consiste na condição de game play. Mesmo que o mundo de jogo de Minecraft possa ser rico e vasto, seu desejo de explorar o mundo é limitado na medida em que ela precisa primeiro garantir a continuação do jogo mantendo seu avatar seguro.

A ideia de Leino de "jogar com um jogo" lembra "a materialidade do jogo"/"the materiality of the game". (LEINO, 2010, p. 141) Ele explica isso com o modo train table (mesa de trem) de Railroads de Sid Meier (Firaxis Games, 2006) que é comparável ao modo criativo de Minecraft. Quando ativado, suspende a condição de game play. Nesse modo, o jogador pode agir livremente como ele quiser dentro do espaço de possibilidades do jogo e não precisa prestar atenção às condições de game play. No modo de game play padrão, o jogador só pode criar quantas faixas seu orçamento permitir, uma limitação imposta pela materialidade do jogo/ sua condição de game play. No modo train table isso não se aplica. No entanto, nem sempre há um modo train table em muitos jogos. Nesses jogos, a condição de possibilidade de jogar com o jogo e finalmente, de experimentar um jogo é, consequentemente, dominar o jogo de tal ponto que as limitações impostas pela materialidade do jogo podem ser superadas e não possuem o mesmo efeito limitador que 
ele poderia ter para um jogador novato. Por outro lado, pode-se jogar com um jogo sem respeitar os limites impostos pela materialidade do jogo, Por exemplo, um explorador pode aceitar possíveis excessos de jogo e simplesmente ignorar a condição de game play. No caso de um game over, ela poderia recomeçar com o salvamento mais recente do jogo.

No nível do game play, agora é possível distinguir entre jogar um jogo de acordo com as condições de game play (game play padrão) e jogar com o jogo, experimentando com a condição de game play através de game play inovador e autêntico (game play como experimentação). Uma segunda forma de jogar com um jogo seria experimentar o jogo no nível da sua estrutura de script e codificação. Isso é possível com editores de jogos que são desenvolvidos por jogadores dedicados ou fornecidos pelo desenvolvedor de jogos. Editores permitem abrir jogos fechados (no sentido Flusseriano) no nível da estrutura do jogo.

\section{Quatro maneiras pelas quais jogos de computador podem ser considerados como sistemas de experimentação ou participar em sistemas de experimentais}

Com essa exposição de jogos de computador como sistemas experimentais - desde que esses sejam jogador em um game play inovador e autêntico em jogos que são complexos o bastante para permitir que a intenção não intencional ocorra - segue-se que existem quatro casos diferentes, nos quais jogos de computador tornam-se verdadeiros geradores de surpresa e, por isso, qualificam-se como sistemas experimentais ou participam de sistemas experimentais.

\subsection{Glitches tornam jogos temporariamente em sistemas experimentais}

Dois desses casos já foram elaborados de passagem. 0 primeiro caso diz respeito a exploração de glitches como exemplificado no exemplo do rocket-jumping em Quake III Arena. Um glitch pode ser considerado uma "abertura" temporária, levando a uma "imprecisão" temporária do artefato de jogo, que permite descobrir uma nova e surpreendente maneira de jogar o jogo. Assim que esse estilo de jogo se torne uma maneira padrão de jogar, o estilo de game play mudou de uma coisa epistêmica para um método estabilizado ou um objeto técnico nos termos de Rheinberger ou uma regra de habilidade. 
(SUITS, 2005, p. 37) Portanto, desse modo o truque do rocket-jumping se torna uma abordagem padrão em lidar com a condição de game play e se torna parte do universo de game play. A maioria dos jogadores, entretanto, nunca experimentou essa abertura em primeira pessoa. Para eles, jogar Quake III Arena já vem com o truque do rocket-jumping como uma regra interna de habilidade. Para eles, eventualmente, é apenas descobrir como realizar esse método o transforma em "coisa oculta" (RHEINBERGER, 1997) e um "desafio kinestésico". (KARHULAHTI, 2013) Nos termos do modelo de expertise de game play ludológico existencial em três camadas de Möring e Leino, o jogador, que descobre o truque do rocket-jumping, desempenha um game play autêntico. No entanto, a vez dos próximos jogadores apenas a experienciam como um game play não autêntico.

\subsection{Jogos complexos e abertos que permitem a intenção não intencional}

O segundo caso diz respeito a jogos de computadores que possuem complexidade suficiente e são capazes de produzir inovações por seus próprios meios (sem bugs ou glitches). Tais jogos, foram descritos como jogos de emergência por Juul. Argumentei anteriormente que esses deveriam ser considerados jogos um nível mais alto de emergência do que o nível 1, como sugerido por Fromm. Esses jogos contêm alto nível de contingência que permite a eles serem geradores de surpresas. Certamente, Minecraft é um exemplar perfeito para esse caso. Ele permite maquinas de engenharia que não foram originalmente planejadas pelos game designers. No entanto, os game designers criaram um jogo que dá permissão para essa intenção não intencional. Considere também Kerbal Space Program. A evidência para esse jogo ser um enorme gerador de surpresas é a grande quantidade de vídeos no Youtube produzidos por entusiastas da física, astronomia, computação voltada para jogos, etc apresentando muitas formas diferentes nas quais aviões e foguetes podem ser projetados. Com frequência, esses veículos voadores são testados no modo criativo do jogo antes de serem implementados no modo de carreira e, portanto, sujeitos à condição de game play. Se o meu foguete explodir no modo criativo, não há nenhum efeito no orçamento da agência espacial que o jogador está configurando no modo carreira de Kerbal Space Program. Normalmente, cada falha afeta o orçamento e as futuras possibilidades para o que o jogador pode fazer no jogo. Como em Minecraft, a complexidade e abertura de Kerbal Space Program levaram a comunidade de jogadores a desenvolver uma Wiki na qual essas novas descobertas são continuamente descritas. Cada modo criativo faz mais sentido à luz 
da existência do modo de sobrevivência em Minecraft ou o modo de carreira em Kerbal Space Program, ambos com uma condição de game play. Os modos criativos são para o modo de condição de game play o que um ambiente de um laboratório científico é para a aplicação e experimentação de um conhecimento científico recém adquirido em uma determinada sociedade. No entanto, os modos criativos em Minecraft e Kerbal Space Program equivalem a um laboratório sem limitações orçamentarias. Entretanto, novas descobertas sobre como jogar o modo carreira em Kerbal Space Program se estabilizarão e se tornarão objetos técnicos, ocultos para serem encontrados por jogadores iniciantes. Para entender o segundo caso, é útil destacar os ciclos de vida dos jogos de computadores contemporâneos, de acordo com os quais os jogos de computador aparecem como processos contínuos e alteráveis, em vez de objetos fixos. (AARSETH, 2001; TAYLOR, 2006; MALABY, 2007; CALLEJA, 2011, p. 910) De acordo com Malaby, "todo jogo é um processo em curso. A medida que é jogado, ele sempre contem potencial para gerar novas práticas e novos significados, possivelmente reconfigurando o jogo em si.". (Ibidem, 2007, p. 102) Essa processualidade não está apenas enraizada na cultura de desenvolvimento de jogadores, mas também no fenômeno em curso de desenvolvimento contínuo de jogos após a publicação de um jogo. Isso culmina na tendência atual dos jogos em "acesso antecipado" (early acess) que são publicados e continuamente atualizados, embora ainda estejam em desenvolvimento e não sejam considerados acabados. Minecraft foi inicialmente lançado como "um lançamento em desenvolvimento" em 2009, versão 1.0 foi lançado em 18 de novembro de 2011. (Wikipedia, 2016) 0 jogo teve numerosas alterações e atualizações desde então. Desse modo, o jogo não está nunca é realmente e completamente definido, mas alimentado com nova "imprecisão". O mesmo pode ser dito para Kerbal Space Program. No ramo do desenvolvimento de software, isso é conhecido como "beta permanente". (NEFF e STARK apud O'DONNELL, 2014, loc. 702) Simultaneamente, quando a comunidade de jogos adiciona funcionalidades ao criar modificações de jogos o jogo permanece imprecisa e pode apresentar ao jogador com novidades. Mas, essas novidades não são apenas inovações no sentido de sistemas experimentais se eles não tiverem sido implementados como coisas escondidas no jogo. A parte das modificações e atualizações de designers, Minecraft também acompanha a geração de conteúdo procedural. (SHAKER et al., 2016) Isso significa que partes do mundo do jogo apenas são geradas quando o jogador navega o seu avatar até os limites do mundo de jogo existente. Esse é um modo como uma imprecisão em processo é implementada em um jogo que potencialmente permite que novas surpresas aconteçam. Designers de jogo só podem 
contabilizar a possibilidade de tais surpresas, mas não para a realização de tais surpresas (intencional não intencional).

\subsection{Desenvolvimento de jogo como sistema experimental}

Isso leva ao terceiro caso que considera o processo de desenvolvimento de jogo. Antes de um jogo ser lançado ao público, o artefato de jogo completo - incluindo a questão de como ele deve se comportar, o que ele ser capaz de fazer, etc - é uma coisa desconhecida que requer uma resposta ainda desconhecida no começo do processo de desenvolvimento. Assim, o processo de desenvolvimento de um jogo foi abordado como um sistema experimental pelo antropólogo Casey O’Donnell (2014). Aqui, entretanto, o artefato de jogo desempenha um papel diferente. Visto que ele é um sistema experimental nos dois casos recém descritos, é apenas uma parte do sistema experimental no processo de desenvolvimento de um jogo. Logicamente e idealmente deveria primeiramente ter o status de uma coisa epistêmica que se torna mais e mais estabilizada e refinada antes que se torne um jogo que pode ser expedido. O’Donnell observa: “até que um projeto atinja a produção, e frequentemente mesmo depois disso, quase todos os aspectos do processo de desenvolvimento do jogo devem agir como um sistema experimental. Deve se aberto ou capaz de fornecer respostas desconhecidas". (O’DONNELL, 2014, p.1153-1154) Em seu livro O’Donnell analisa as estruturas, os estágios, as práticas, as tecnologias, os padrões, os papéis (p. exp. ferramentas de engenharia, artistas técnicos, engenheiros, e game designers) etc do processo de produção (ibidem, 2014, loc. 481-482) que juntos formam o sistema experimental. Sem entrar em muitos detalhes com a análise de O'Donnell pode-se imaginar que mesmo um jogo aberto e intencionalmente vago pode se tornar uma coisa epistêmica e, eventualmente, o objetivo de um processo de produção. Como é então possível pensar em jogos continuamente abertos, como Minecraft ou Kerbal Space Program, como sendo o resultado desejado do processo de desenvolvimento de um jogo, se os próprios jogos são sistemas experimentais em potencial? Intuitivamente, pode-se supor que um jogo de computador expedível é um objeto suficientemente estabilizado (técnico) da mesma forma que Limbo oferece o mesmo comportamento de game play repetitivamente, que recria de forma confiante e repetida a mesma maneira de ser jogado em um pequeno espaço de possibilidade e desvio. 0 artefato de jogo fornece respostas conhecidas quando é 
transformado em dispositivo de teste antes de ser desviado. No entanto, existem também franquias de jogos, como a série Assassin's Creed (Ubisoft Montreal, 2007), que repetidamente produzem novas instâncias de um jogo, adicionando uma pequena quantidade de novos recursos a cada nova interação do jogo. Essa é uma forma um pouco diferente de como um jogo pode ser considerado um beta permanente. Cada instância do jogo é um objeto técnico em si que mesmo que se abre para cada versão sucessiva e requer novos processos interativos de desenvolvimento de jogos e testes de jogo (cf. FULLERTON, 2008, p. 248-276) até que a nova coisa epistêmica seja descoberta e suficientemente estabilizada. Para um jogo complexo e aberto como Kerbal Space Program deve-se presumir que sua abertura contínua deriva de um grande espaço de possibilidade, mas simultaneamente uma capacidade contínua de fornecer respostas para questões desconhecidas; sendo esse último o real objetivo do processo de produção. Entretanto, partes de um jogo também precisam ser suficientemente estabilizadas para serem identificáveis como o Kerbal Space Program e não algum outro jogo. Desse modo, tais jogos contêm um paradoxo de estabilidade e instabilidade ou imprecisão e certeza. Além disso, o terceiro caso incorpora o primeiro e o segundo casos, ou versões deles, durante a fase de playtesting de projeto interativo. Quando o game design é implementado em um protótipo de jogo e se torna o playtesting, os desenvolvedores de jogo conhecem seu sistema e decidem qual características eles pretendem estabilizar. Esses elementos do artefato do jogo são elemento do jogo que se qualificam como dispositivos de teste. Portanto, se considerarmos os casos, 1, 2 e 3 como níveis diferentes do processo de game design, as partes do dispositivo de teste devem ser consideradas o nível zero. No segundo nível, os designers descobrem quais bugs e glitches podem ser transformados em "recursos". Eventualmente, para um jogo aberto como Kerbal Space Program, desenvolvedores decidem quais elementos no artefato de jogo permanecem no nível do dispositivo de teste e permitir a discernibilidade do artefato de jogo, quais características abertas e contingentes eles implementam, e até que ponto o jogo permanece aberto para permitir futuras respostas desconhecidas. Jogos compreendidos como beta permanente estão em constante "mudança e fluxo" (O’DONNEL, 2014, loc.706), constantemente oscilando entre objetos técnicos e coisas epistêmicas. Um jogo como Limbo, entretanto, não deve chegar ao terceiro nível apenas através de game play, em oposição a Kerbal Space Program e ao Minecraft. 


\subsection{Jogos como objetos técnicos de sistemas experimentais matemáticos}

O quarto caso reduz ainda mais e considera os jogos de computador como a) resultantes de sistemas experimentais de outros campos de conhecimento e b) sistemas experimentais derivando de jogos, mas que influenciam outros campos do conhecimento. Em oposição aos primeiros três casos que focam a extensão do game play como o resultado de um sistema experimental, esse caso se refere a um contexto mais amplo sobre a relação dos jogos de computador com o conhecimento fora de um artefato de jogo individual. Em sua tese, o filósofo da mídia Alemã, Claus Pias (2000) segue o argumento de que jogos de computador ["que requerem um computador" /“which require a computer" (Pias 2000, 6)] requerem certos tipos e formações de conhecimentos antes que houvesse a possibilidade de se tornem coisas epistêmicas em potencial, um objetivo de descoberta em um processo experimental e uma coisa real. Enquanto Pias identifica três tipos ideais de jogos de computador (jogos de estratégia são críticos na configuração, jogos de aventura decisivos, e jogos de ação são críticos no tempo), particularmente jogos de ação requerem conhecimento de campos como psicologia experimental com seus métodos de "mensuração de capacidades sensorimotoras", "funcionalismo e behaviorismo" com seus "testes de aprendizado e comportamento", "ciências do trabalho" e suas "padronizações de opções de ações, bem como o sequenciamento de tempo e espaço" (por exemplo, quando se trabalha com a linha de montagem) e a "visibilidade e comensurabilidade do computador" ("visibility and commensurability of the computer"). (PIAS, 2002, p. 11) Paradoxalmente, os campos cujo conhecimento, tecnologias, e práticas permitem a possibilidade de jogos de ação trazem simultaneamente o que Rheinberger se refere como dispositivos de teste. A anedota de como um dos primeiros jogos de computador surgiu é um exemplo de um sistema experimental diferente que produziu um produto paralelo conhecido hoje como Tennis for Two (Higinbotham, 1958). É amplamente conhecido que Tennis for Two é o resultado da necessidade do Laboratório Nacional Brookhaven [Brookhaven National Laboratory's (BNL)] de demonstrar seu trabalho ao público no "Dia do Visitante". 0 inventor de Tennis for Two, William Higinbotham, costumava desenvolver sistemas de ataque ar-terra aos Bombardeios B-29 (Pias erroneamente se refere aos Bombardeios B-28) e ao ProjetoManhattan antes de começar a trabalhar na BNL onde ele estava preocupado com o "o impacto civil na tecnologia nuclear". (PIAS, 2000, p. 9) Ante ao problema pragmático sobre 
como demonstrar a pesquisa em "balística e cronometragem" para audiência, Higinbitham usou o computador do laboratório e o osciloscópio para criar o Tennis for Two com a finalidade de tornar o trabalho da BNL visível e compreensível. (PIAS, 2000, p. 9-10) Consequentemente, um dos percursores mais proeminentes dos jogos de computador de ação foi em si mesmo uma surpresa e uma resposta a uma questão desconhecida derivada de um sistema experimental planejado para calcular problemas de balística e cronometragem.

Enquanto o Tennis for Two resultou de um sistema experimental designado para física, von Reißwitz' Kriegsspiel foi parte de um sistema experimental que avançou o conhecimento de "confrontos de batalha e matemática". (HILGERS, 2012, p. ix) 0 livro de Phillip von Hilgers, War Games (2012), demonstra que outro ancestral direto dos jogos de computador contemporâneos, o Kriegsspiel (uma simulação de campo de batalha entre dois oponentes apresentando um tipo de jogador neutro cuja tarefa é computar os resultados dos movimentos dos participantes) dos generais prussianos do começo do século XIX. O jogo em si foi desenvolvido com o objetivo de ensinar e avaliar táticas de guerra. 0 Kriegsspiel pode ser considerado um objeto técnico dentro de um sistema de experimentação devotado a coisa epistêmica a respeito de como ganhar vantagens militares no campo de batalha. A comparação entre cenários reais de batalha e a capacidade do jogo de representar de forma confiável esses cenários leva a gerar o Kriegsspiel como objeto técnico de volta para o estágio de uma coisa epistêmica com o objetivo de estabilizá-lo novamente em um objeto técnico responsável por novos conhecimentos adquiridos no campo de batalha. Desse modo, pode-se dizer que Kriegsspiel esteve em um beta permanente enquanto foi usado para treinar generais e para levar a táticas úteis no campo de batalha e até mesmo com o propósito de entretenimento com o novo jogo de guerra que por sua vez foi usado novamente por exemplo para calcular estratégias relacionadas a invasão do Kuwait na década de 1990. (cf. HILGERS, 2000) Para o propósito desse artigo, posso apenas fornecer um relato muito reducionista do argumento de von Hilgers e peço a todos que quiserem refazê-lo para recorrer ao livro de von Hilgers. O Kriegsspiel não foi apenas instrumental para ganhar conhecimento em estratégicas e táticas em guerra, em combinação com conhecimento de teoria de guerra ou por exemplo Carl von Clausewitz que historicamente participou do avanço da matemática. Von Hilger aponta que entre outras formações de conhecimento um dos problemas centrais da teoria de guerra de von Clausewitz foi: 
"as fricções da guerra e o "nevoeiro da guerra" que o levaram a rejeitar o postulado da calculabilidade geral. Ao fazê-lo, ele esboçou explicitamente um conceito de probabilidade [...], que primeiro se tornaria uma ferramenta epistemológica da matemática e da física com a termodinâmica”. (HILGERS, 2012, p. x).

Enquanto "a doutrina de Clausewitz antecipa esse desenvolvimento numa veia teórica e compreende a necessidade de lidar com a contingência em cenários de guerra, "o poder de comando é realmente implementado pela primeira vez no meio do jogo de guerra tático". (HILGERS, 2012, p. xi) George Leopold von Reißwitz desenvolveu o jogo de Guerra tático (taktisches Kriegsspiel) no começo do século XIX. Quando Kriegsspiel é colocado em uso, a contingência se torna uma "dupla contingência": uma vez no nível do artefato de jogo e novamente na situação real de guerra para qual o jogo é usado para examinar a melhor estratégia. Von Hilgers então conclui que o conhecimento produzido nesse processo leva a teoria de jogo de Neumann que tem um grande impacto na calculabilidade da guerra, mas também no modo como a contingência pode ser calculada em geral. (HILGERS, 2012, p. xii) É a esse respeito que o Kriegsspiel foi o primeiro uma coisa epistêmica resultando entre outras coisas a teoria de guerra de Clausewitz e que alimenta de volta como um objeto técnico no desenvolvimento da teoria matemática. Ironicamente, isso se tornou conhecido como a teoria dos jogos para calcular contingência.

\section{Conclusão}

Esse artigo fornece uma compreensão diferente da experimentação em game play de computador que permite distinguir entre o game play padrão que é frequentemente compreendido com uma noção de senso comum de experimentação e experimentação no sentido de sistema experimental. Isso torna possível identificar jogos de computador que se qualificam como meros dispositivos de teste cujo game play foi até agora descrito como experimentação.

Tem sido argumentado que uma certa abertura nos jogos é uma condição necessária para que eles sejam potencialmente sistemas experimentais. Essa abertura pode ocorrer diferentes maneiras: os jogos são abertos porque são sistemas que permitem um alto nível de emergência, podem ser abertos porque contêm bugs ou glitches, e estão abertos porque estão em beta permanente. Além dessa abertura, esses jogos também exigem um tipo de 
jogador inovador que jogue um jogo de forma autentica ou em um modo próprio, pois ela transcende estilos existentes e já descobertos de game play de um determinado jogo.

Apesar de um certo grau de abertura e imprecisão, os jogos abertos precisam ter elementos estabilizados que respondam por sua identidade e discernibilidade, enquanto reconhecem a possibilidade de que os modos de como eles são jogados possam potencialmente mudar o tempo.

Os quatro casos discutidos de como os jogos são ou participam de sistemas experimentais podem ser considerados como quatro camadas de experimentação que são hierárquicas e diferentes em grau e não em qualidade. A camada mais baixa seria jogos tão estabilizados que se qualificam como dispositivos de teste. A camada acima consiste em jogos que são em sua maioria estabilizados, mas contêm bugs ou glitches que levam a uma abertura imprecisão temporária que podem levar a novas maneiras de jogá-los. A camada seguinte consiste em jogos de computador que são tão complexos e abertos que permitem a intencionalidade não intencional do game play. Essas três camadas estão contidas no processo de desenvolvimento de jogos de computador. Não importa quais tipos de jogos os desenvolvedores pretendem produzir em seus projetos interativos, eles precisam passar pelo singular ou por todas as três camadas inferiores repetidamente para descobrir a coisa epistêmica de seu processo de desenvolvimento. Eventualmente, há a mais alta ordem na qual os jogos de computador resultam de novos conhecimentos de diferentes domínios de conhecimento ou que ajudam a descobrir novos conhecimentos em domínios de conhecimento que não relacionados a jogos. A camada final mostra como as práticas de conhecimento em níveis muito baixos de jogos de computador são eventualmente contidas em culturas de conhecimentos maiores, se jogos específicos são relevantes para eles. Isso, entretanto, é histórico e não conta para cada jogo de computador.

As descobertas desse artigo são relevantes para os domínios da hermenêutica dos jogos, uma vez que abordar os jogos de computador pela teoria dos sistemas experimentais permite distinguir diferentes jogos de computador pelo seu potencial de trazer novos conhecimentos, embora esse conhecimento seja relacionado principalmente ao conhecimento do jogo em si. Acredito que esses achados também são de interesse para o domínio de pesquisa que investiga jogos e aprendizado, uma vez que mostra que os jogos nem sempre criam conhecimento apenas por meio de seu design. Parece mais importante que eles estejam inseridos em culturas de pesquisa mais amplas que, provenientes de uma 
história específica do conhecimento, fazem uso de jogos em um determinado ponto de sua trajetória de investigação.

\section{Referências Bibliográficas}

AARSETH, Espen. Cybertext: Perspectives on Ergodic Literature. Baltimore: Johns Hopkins University Press, 1997.

AARSETH, Espen. Computer Game Studies, Year One. Game Studies: The International Journal of Computer Game Research, [s.l], v. 1, n. 1, jul. 2001. Disponível em: http://www.gamestudies.org/0101/editorial.html. Acesso em: 04 abr. 2019.

AARSETH, Espen. Playing Research: Methodological approaches to game analysis. In: GAME APPROACHES / SPIEL-VEJE. PAPERS FROM SPILFORSKNING.DK CONFERENCE, 2003, Melbourne. Proceedings... . Melbourne: 2003. p. 1 - 7.

AARSETH, Espen. I fought the law: Transgressive play and the implied player. In: DIGRA, 2007, [s.l]. Proceedings... . [s.l]: 2007. p. 25 - 28.

ARJORANTA, Jonne. Do We Need Real-Time Hermeneutics?: Structures of Meaning in Games. In: DIGRA, 5., 2011, Hilversum. Proceedings... . Hilversum: Digra e Utrecht School Of Arts, 2011. p. 1 - 1. Disponível em: http://www.digra.org:8080/Plone/dl/display_html?chid=11310.17396.pdf. Acesso em: 04 abr. 2019.

ARJORANTA, Jonne. Real-Time Hermeneutics: Meaning-Making in Ludonarrative Digital Games. 2015. Tese (Doutorado) - University Of Jyväskylä, Jyväskylä, 2015.

ARSENAULT, Dominic; PERRON, Bernard. In the Frame of the Magic Cycle: The Circle(s) of Gameplay. In: PERRON, Bernard; WOLF, Mark J. P. (Ed.). The Video Game Theory Reader 2. New York: Routledge, 2009. p. 109-133.

BARTLE, Richard. Hearts, Clubs, Diamonds, Spades: Players who suit MUDs. 1996. Disponível em: http://mud.co.uk/richard/hcds.htm. Acesso em: 04 abr. 2019.

CALLEJA, Gordon. 2011. In-Game: From immersion to incorporation. Cambridge, MA: MIT Press. 2011.

DREYFUS, Hubert. Being-in-the-world: a commentary on Heidegger's Being and time, division I. Cambridge, MA: MIT Press. 1991.

FLUSSER, Vilém. Kommunikologie. 1. Aufl. Frankfurt: Fischer Taschenbuch Verlag, 1998.

FROMM, Jochen. Types and Forms of Emergence. 2005. Disponível em: https://arxiv.org/ftp/nlin/papers/0506/0506028.pdf. Acesso em: 04 abr. 2019.

FULLERTON, Tracy. Game Design Workshop: A Playcentric Approach to Creating Innovative Games. 2nd ed. Amsterdam, Boston: Elsevier Morgan Kaufmann. 2008. 
GALLOWAY, Alexander. Countergaming. In: GALLOWAY, Alexander. Gaming: essays on algorithmic culture. Minneapolis: University of Minnesota Press, 2006. p. 107-126.

HEIDEGGER, Martin. Being and time. New York: HarperPerennial/Modern Thought, 2008.

HILGERS, Philipp von. Eine Anleitung zur Anleitung. Das taktische Kriegsspiel 1812-1824.

Board Game Studies, n. 3, p. 59-77, 2000.

HILGERS, Philipp von. War games: a history of war on paper. Cambridge, MA: MIT Press. 2012.

JUUL, Jesper. The Open and the Closed: Games of Emergence and Games of Progression. In: DIGRA, 2002, Tampere. Proceedings.... . Tampere: Tampere University Press, 2002. p. 323 329.

JUUL, Jesper. Half-Real: Video Games between Real Rules and Fictional Worlds. Kindle edition. Cambridge: MIT Press, 2005.

KARHULAHTI, Veli-Matti. Double Fine Adventure and the Double Hermeneutic Videogame: Fun and Games'. Toulouse, 2012.

KARHULAHTI, Veli-Matti. Adventure games in kinesthetic videogame theory: A demandbased framework for conceptualizing the narrative. In: DOCTORAL CONSORTIUM AT FOUNDATIONS OF PLAY AND GAMES CONFERENCE, 2013, Chania. Proceedings... . Chania: 2013.

KARHULAHTI, Veli-matti. Adventures of Ludom: : A Videogame Geneontology. 2015. $1 \mathrm{f}$. Tese (Doutorado) - University Of Turku, Turku, 2015.

KRPATA, Mitch. Limboned. 2010. Disponível em: http://insultswordfighting.blogspot.com/2010/08/limboned.html. Acesso em: 04 abr. 2019.

LEINO, Olli Tapio. Understanding games as played: Sketch for a first-person perspective for computer game analysis. In: THE PHILOSOPHY OF COMPUTER GAMES CONFERENCE, 1. , 2009, Oslo. Proceedings... . Oslo: University Of Oslo, 2009.

LEINO, Olli Tapio. Emotions in play: On the constitution of emotion in solitary computer game play. Tese (Doutorado) - IT University of Copenhagen, Copenhagen, 2010.

LEINO, Olli Tapio. Death Loop as a Feature. Game Studies: The International Journal of Computer Game Research, [s.l], v. 12, n. 2, 2012. Disponível em:

<http://gamestudies.org/1202/articles/death_loop_as_a_feature>. Acesso em: 04 abr. 2019.

LEINO, Olli Tapio. Who should I call if no one shows up to pick up the dead? \#movingout": On gameness, materiality, and meaning in Cities: Skylines. In: THE PHILOSOPHY OF COMPUTER GAMES CONFERENCE, 2015, Berlim. Proceedings... . Berlim: 2015. 
LEINO, Olli Tapio; MÖRING, Sebastian. Authenticity Quest: On the conditions of possibility for 'being yourself' in a computer game. In: DIGRA, 2015, Lüneburg. Proceedings... . Lüneburg: 2015

LINDEROTH, Jonas. Beyond the digital divide: An ecological approach to gameplay.

Transactions of the Digital Games Research Association, v. 1, n. 1, 2013. Disponível em: http://todigra.org/index.php/todigra/article/view/9. Acesso em: 04. Abr. 2019.

MALABY, Thomas M. Beyond Play: A New Approach to Games. Games and Culture v.2, n. 2, p. 95-113, abr. 2007.

MCCORMICK, Rich. Spaceships worth more than $\$ 200,000$ destroyed in biggest virtual space battle ever. The Verge. [s.l], jan. 2014. Disponível em:

<https://www.theverge.com/2014/1/29/5356498/eve-online-battle-sees-200000-dollarsworth-of-spaceships-destroyed>. Acesso em: 04 abr. 2019.

MÖRING, Sebastian. Games and Metaphor - A critical analysis of the metaphor discourse in game studies. Copenhagen: IT University of Copenhagen. 2014. Disponível em:

http://bit.ly/1Gtcg4k. Acesso em: 04. Abr. 2019.

O'DONNELL, Casey. Developer's dilemma: the secret world of videogame creators. Inside technology. Cambridge, Massachusetts: The MIT Press. 2014.

OXFORD UNIVERSITY. exploration. In: OXFORD UNIVERSITY. Oxford english

Dictionary. Oxford: Oxford University Press, 2016. p. 1-1. Disponível em:

<http://www.oed.com/view/Entry/66662>. Acesso em: 04 abr. 2019.

PEARCE, Celia. Sims, BattleBots, Cellular Automata God and Go. A Conversation with Will Wright by Celia Pearce. Game Studies. v. 2, n. 1, 2002. Disponível em:

http://www.gamestudies.org/0102/pearce/. Acesso em: 04 abr. 2019.

PIAS, Claus. Computer Spiel Welten. Tese (Doutorado) - Bauhaus University Weimar, Weimar, 2000. Disponível em: http://e-pub.uni-weimar.de/volltexte/2004/37/. Acesso em 04 abr. 2019.

PIAS, Claus. Computerspiele. 2002. Disponível em: http://www.unidue.de/ bj0063/texte/k+u.pdf. Acesso em: 04 abr. 2019.

RHEINBERGER, Hans-Jörg. Toward a history of epistemic things: Synthesizing proteins in the test tube. Stanford, MA: Stanford University Press. 1997.

RHEINBERGER, Hans-Jörg. Experimental Systems, Graphematic Spaces. In: LENOIR, Timothy (Org.). Inscribing Science: Scientific Texts and the Materiality of Communication. [s.l]: Stanford University Press, 1998. p. 285-303.

SALEN, Katie; ZIMMERMAN, Eric. Rules of Play: Game Design Fundamentals. Kindle edition. Cambridge, MA: MIT Press, 2004.

SHAKER, Noor; TOGELIUS, Julian; HRSG, Mark Nelson. Procedural content generation in 
games. New York: Springer, 2016.

SUITS, Bernard. The Grasshopper: Games, Life and Utopia. [s.l]: Broadview Press, 2005.

TAYLOR, T.L. Play between worlds: Exploring online game culture. Cambridge, MA; London: MIT Press, 2006

VELLA, Daniel. The Ludic Subject and the Ludic Self: Analyzing the 'I-in-the-Gameworld'. Copenhagen: IT University of Copenhagen, 2015.

WIKIPEDIA. Minecraft. Wikipedia. Disponível em: https://en.wikipedia.org/w/index.php?title=Minecraft\&oldid=746162291. Acesso em: 04 abr. 2019.

\section{Referências Ludográficas}

CCP Games. 2003. EVE Online. CCP Games.

Firaxis Games. 2006. Sid Meier's Railroads! [Windows]. 2K Games.

HIGINBOTHAM, William. 1958. Tennis for Two. [Analgo computer, oscilloscope].

id Software. 1999. Quake III Arena. [Microsoft Windows]. Activision.

Keen Software House. 2013. Space Engineers. [Windows PC]. Keen Software House.

Mojang. 2011. Minecraft. [Windows PC et.al.]. Mojang.

Playdead. 2010. Limbo. [Xbox 360; PlayStation 3; Windows; Mac OS; Linux; iOS; PlayStation Vita]. Copenhagen, Denmark: Microsoft Game Studios; Playdead.

Squad. 2015. Kerbal Space Program. [Windows PC]. Squad.

Ubisoft Montreal. 2007. Assassin's Creed. [Xbox 360; PlayStation 3, Windows]. Ubisoft.

\section{Understanding single player computer games as experimental systems}

\footnotetext{
Abstract:

This paper analyzes and theorizes the conditions which allow to understand single player computer game play (SPCGP) as part of a knowledge production practice called experimentation. It therefore draws on Hans-Jörg Rheinberger's theory of the experimental system. This approach is a useful contribution to
} 
the field of computer game hermeneutics as it allows to understand game play as a hermeneutic process as well as describing the hermeneutic game play circle in more detail. The paper argues, that so far experimentation in game studies and game hermeneutics has been equated with standard game play processes. In other words, all game play is experimentation. The examples often mentioned in those cases are, however, mere testing devices according to Rheinberger's theory. In order to be an experimental system, computer games themselves have to be capable of producing true novelties. This depends, on the one hand, on the level of complexity a given game exhibits and, on the other hand, it depends on the degree of creativity of a given player. Eventually the paper suggests that there are only four different cases in which computer games become true generators of surprises and thus qualify as or participate in experimental systems which I will present and discuss: glitches in games which facilitate so-called exploits in SPCGP, sufficiently complex games with a high degree of emergence, the game production process, and computer games which have historically participated in the advancement of science.

\section{Keywords:}

Computer game play. Single player. Experimental system. Hermeneutics. Testing device. 